\title{
Editorial: Machine learning for safety-critical applications in engineering
}

\author{
Mariam Kiran ${ }^{1}$ (D) Samir Khan ${ }^{2}$
}

(c) The Author(s), under exclusive licence to Springer Science+Business Media LLC, part of Springer Nature 2020

As our society becomes more automated and technology efficient, the need to implement safe engineering solutions become paramount. Whilst real-time machine learning is being championed to remove any technological barriers, its successful realisation is inevitably hindered by computational requirements, model quality assurance and justification of resource costs. Yet, due to recent industrial drive, the field is moving quickly with unprecedented interest from several technology organisations, academic disciplines, and even the general public. Machine learning is enabling an array of real-world commercial products and services that were not technologically feasible before and hence can prove useful for safety-critical applications.

This special section is based on two papers presented during a workshop session at the thirty-fifth International Conference on Machine Learning (ICML 2018) on Machine Learning for Safety-Critical Applications in Engineering. The workshop had focused on bridging research communities from Artificial Intelligence (AI) and engineering, whilst encouraging developments that support practical solutions for safety-critical applications. Out of the 12 papers presented at the workshop, 6 papers were invited to submit significantly enhanced research. Following further peer review, 2 papers have been finally accepted for this special section. These papers reflect the latest research and methods of using machine learning to deal with open problems in engineering systems and anomalies during network transfers. The first paper titled 'Engineering Problems in Machine Learning', emphasises on establishing quality assurance frameworks for machine learning approaches in automated driving systems. The second paper titled 'Detecting Anomalous Packets in Network Transfers', demonstrates the importance of unsupervised feature classification methods for networking applications.

Both papers advocate that even though machine learning can add business value to many engineering communities, its adoption must be realised through a framework that should be scrutinised by field experts. With the increasing use of black-box models, where most confirmations are empirical rather than a mathematical solution to a learning problem, the implicit trust would decrease. As a result, even if the AI-based reasoner may seem more accurate

Mariam Kiran

mkiran@es.net

Samir Khan

khan@ailab.t.u-tokyo.ac.jp

1 Lawrence Berkeley National Laboratory, Berkeley, CA 94720, USA

2 University of Tokyo, Tokyo 113-8654, Japan 
compared its conventional engineering counterpart, its lack of explain ability eventually leads to a lack of trust in the outcome. The black-box results need to reveal the full decision-making process that is comprehensible to humans. This promotes more transparency and verification of AI-based solutions if they are to be used for a safety-critical application.

Publisher's Note Springer Nature remains neutral with regard to jurisdictional claims in published maps and institutional affiliations. 